\title{
Controlling inflammation by selective autophagy
}

\author{
Junghyun Lim ${ }^{1} \cdot$ Aditya Murthy (iD) $^{1}$
}

A complex interaction between host genetics, commensal and pathogenic microflora, inorganic and organic environmental stresses (e.g., chemical toxins, pharmaceuticals, diet) combine to direct the onset as well as progression of chronic inflammatory diseases. Our understanding of inflammatory bowel disease has benefited greatly from the advent of genome-wide association studies (GWAS). In addition to confirming the role of established pathways such as pathogen sensors, innate signaling adapters, and cytokines/ receptors, GWAS has identified significant associations between defects in cellular homeostatic processes such as autophagy or the ER stress response and increased risk of disease [1]. Indeed, defects in autophagy have been implicated in numerous inflammatory pathologies including lupus, rheumatoid arthritis, and neurodegeneration [2-4].

Autophagy directs the breakdown of intra-cellular cargo via lysosomal degradation and represents one of the two major pathways regulating protein homeostasis, the other being the ubiquitin-proteasome system. A Thr-300-Ala (T300A, rs2241880) missense variant in the key autophagy adapter gene Atg1611 was identified as a consistent risk allele for Crohn's disease by Hampe et al. [5], and numerous studies following this observation generated consistent findings with Atg1611 gene deletion or hypomorph alleles in murine models [6, 7]. The molecular consequence of the T300A variant was revealed by two independent studies identifying that this amino acid change resulted in enhanced Caspase-mediated degradation of human and murine ATG16L1 [8, 9]. While phenotypes of enhanced inflammation upon deletion of core autophagy genes continue to be described, molecular mechanisms underlying these observations have remained unresolved. In addition, how inflammatory signaling is terminated when the initial stimulus is dissipated has yet to be fully elucidated. Does defective autophagy confer a generalized "proinflammatory" fate upon the cell and organism, or are

Aditya Murthy

murthy.aditya@gene.com

1 Department of Cancer Immunology, Genentech, Inc., 1 DNA Way, South San Francisco, CA 94080, USA particular inflammatory signaling nodes more susceptible to regulation by autophagy? Is this effect evoked by accumulating pro-inflammatory proteins that may be regulated by autophagy, leading to uncoordinated inflammatory responses? We posed this question in the context of Tolllike receptor (TLR) signaling [10], as this pathway is central to innate host defense and has been extensively characterized in the context of its major adapters TRIF and MYD88 [11]. Whereas MYD88 functions downstream of the majority of TLRs, TRIF (encoded by the gene Ticam1) lies downstream of TLR3 and 4 and can potentiate cell death as well as an inflammatory cytokine response [12].

An initial comparison of cytokine secretion by murine bone marrow-derived macrophages (BMDMs) following TLR stimulation revealed that loss of ATG16L1 specifically elevated Type I interferon (i.e., IFN- $\beta$ ) upon TLR3 or TLR4 engagement. Deletion of another core autophagy gene, Atg5 conferred consistent phenotypes, indicated that autophagosome biogenesis and its extension was important in the regulation of TLR3/4-mediated signaling. Characterization of TLR4 signaling confirmed that the TRIF-TBK1-IRF3 axis was overactive in ATG16L1-deficient BMDMs, resulting in elevated gene transcription of IRF3 targets such as Ifnbl and a robust enhancement of the interferon response. Bone marrow-derived dendritic cells also exhibited similar phenotypes upon loss of ATG16L1, confirming that this mechanism acted in multiple myeloid-lineage cells participating in the innate response to pathogens.

Both MYD88 and TRIF have been described to form large molecular platforms to precipitate downstream inflammatory signaling, termed the "MyDDosome" or the "TRIFfosome", respectively [11]. Thus, we asked whether loss of ATG16L1 impacted the turnover or stability of these proteins in BMDMs. Strikingly, accumulation of oligomeric forms of TRIF but not MYD88 was observed in ATG16L1-deficient cells in cycloheximide chase assays. These findings prompted a hypothesis that TRIF itself could be cargo for a targeted form of autophagy, namely selective autophagy. Long thought to be a non-selective process, emerging evidence has established that the autophagic machinery can identify cytosolic cargo in a targeted manner. This is accomplished via cargo association with cargo-recognition proteins termed autophagy 


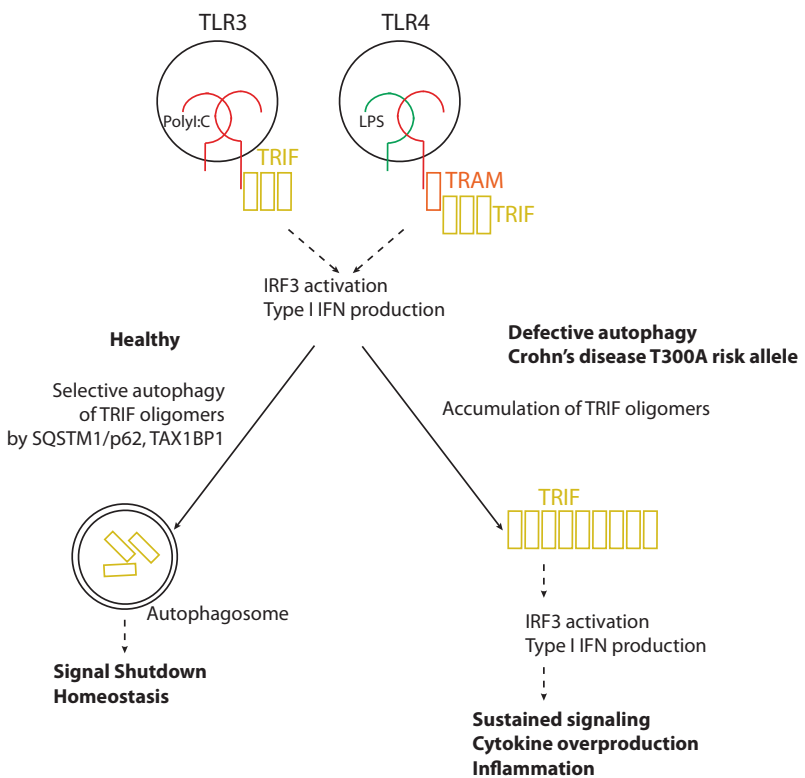

Fig. 1 Regulation of TRIF-mediated signaling by selective autophagy. Engagement of TLR3/4 induces oligomerization of the innate adapter TRIF, which is subsequently targeted by autophagy receptors TAX1BP1 and SQSTM1/p62 for autophagic clearance and return to homeostasis. Defective autophagy sustains the inflammatory signal due to attenuated turnover of TRIF.

receptors, exemplified by selective autophagy of organelles and protein aggregates [13]. We thus undertook a multiplexed tandem mass tagging-based mass-spectrometry approach (TMT-MS) to identify autophagy receptors which may associate with TRIF. This effort revealed two substantial findings: First, that loss of ATG16L1 and thus autophagosomes did not result in a dramatic defect in proteome homeostasis overall. Indeed, less than 50 proteins displayed significantly altered abundances between control and ATG16L1-deficient macrophages. Second, varied kinetics of protein accumulation were made apparent with TMT-MS. We identified distinct proteins enriched at baseline or following TLR4 engagement by ATG16L1-deficient BMDMs. Among these, specific autophagy receptors (i.e., CALCOCO1, FAM134b, FAM134c, SQSTM1/p62, and TAX1BP1) emerged as candidates that bind TRIF. Biochemical assessment of endogenous TRIF revealed TAX1BP1 and SQSTM1/p62 as its binding partners; knockdown of Tax $1 \mathrm{bp} 1$ in wildtype BMDMs conferred a pro-inflammatory phenotype consistent with ATG16L1 deficiency. Thus, TAX1BP1 was identified as a relevant autophagy receptor that regulates TRIF signaling (Fig. 1). Given the elevated production of IFN- $\beta$ by ATG16L1-deficient BMDMs ex vivo, we tested its significance in vivo using models of LPSmediated sepsis as well as enteric infection with virulent S. typhimurium. In both systems, myeloid cell-specific loss of ATG16L1 conferred a hyper-inflammatory phenotype which was rescued by pharmacological blockade of IFNAR1.

Our findings were extended to human immunology, first by assessment of the T300A variant of ATG16L1 using primary human monocyte-derived macrophages. Homozygosity for the T300A genotype resulted in enhanced IFN- $\beta$ production upon TLR3/4 engagement. Next, we observed a sustained interferon gene response signature (IRG) in Crohn's disease and ulcerative colitis patients who failed to respond to anti-TNF therapy. Cumulatively, we and others $[14,15]$ have demonstrated that inhibiting autophagy results in an aberrant pro-inflammatory response via sustained activation of specific signals, providing molecular clues to the type of innate immunity elicited by defects in this critical immunoregulatory pathway.

\section{Compliance with ethical standards}

Conflict of interest J.L. and A.M. are employees of Genentech, Inc., a for-profit organization.

\section{References}

1. Jostins L, et al. Host-microbe interactions have shaped the genetic architecture of inflammatory bowel disease. Nature. 2012;491:119-24. https://doi.org/10.1038/nature11582.

2. International Consortium for Systemic Lupus Erythematosus, G et al. Genome-wide association scan inwomen with systemic lupus erythematosus identifies susceptibility variants in ITGAM, PXK, KIAA1542 and other loci. Nat Genet. 2008;40:204-10. https://doi.org/10.1038/ng.81.

3. Menzies FM, et al. Autophagy and Neurodegeneration: Pathogenic Mechanisms and Therapeutic Opportunities. Neuron. 2017;93:1015-34. https://doi.org/10.1016/j.neuron.2017.01.022.

4. Rockel JS, Kapoor M. Autophagy: controlling cell fate in rheumatic diseases. Nat Rev Rheumatol. 2016;12:517-31. https://doi. org/10.1038/nrrheum.2016.92.

5. Hampe J, et al. A genome-wide association scan of nonsynonymous SNPs identifies a susceptibility variant for Crohn disease in ATG16L1. Nat Genet. 2007;39:207-11. https://doi.org/10.1038/ ng1954.

6. Cadwell K, et al. Virus-plus-susceptibility gene interaction determines Crohn's disease gene Atg16L1 phenotypes in intestine. Cell. 2010;141:1135-45. https://doi.org/10.1016/j.cell.2010.05. 009.

7. Saitoh T, et al. Loss of the autophagy protein Atg16L1 enhances endotoxin-induced IL-1beta production. Nature. 2008;456:264-8. https://doi.org/10.1038/nature07383.

8. Lassen KG, et al. Atg16L1 T300A variant decreases selective autophagy resulting in altered cytokine signaling and decreased antibacterial defense. Proc Natl Acad Sci USA. 2014;111:7741-6. https://doi.org/10.1073/pnas.1407001111.

9. Murthy A, et al. A Crohn's disease variant in Atg1611 enhances its degradation by caspase 3. Nature. 2014;506:456-62. https://doi. org/10.1038/nature13044.

10. Samie M, et al. Selective autophagy of the adaptor TRIF regulates innate inflammatory signaling. Nat Immunol. 2018. https://doi. org/10.1038/s41590-017-0042-6. 
11. Gay NJ, Symmons MF, Gangloff M, Bryant CE. Assembly and localization of Toll-like receptor signalling complexes. Nat Rev Immunol. 2014;14:546-58. https://doi.org/10.1038/nri3713.

12. Blander JM. A long-awaited merger of the pathways mediating host defence and programmed cell death. Nat Rev Immunol. 2014;14:601-18. https://doi.org/10.1038/nri3720.

13. Khaminets A, Behl C, Dikic I. Ubiquitin-Dependent And Independent Signals In Selective Autophagy. Trends Cell Biol. 2016;26:6-16. https://doi.org/10.1016/j.tcb.2015.08.010.
14. Gentle IE, et al. TIR-domain-containing adapter-inducing interferon-beta (TRIF) forms filamentous structures, whose pro-apoptotic signalling is terminated by autophagy. FEBS J. 2017;284:1987-2003. https://doi.org/10.1111/febs.14091.

15. Yang Q, et al. TRIM32-TAX1BP1-dependent selective autophagic degradation of TRIF negatively regulates TLR3/4-mediated innate immune responses. PLoS Pathog. 2017;13:e1006600 https://doi.org/10.1371/journal.ppat.1006600. 